\title{
Power Amplifiers for Next Generation Wireless Platforms
}

\author{
Kevin Tom, Vandana Basoo, Mike Faulkner, *Thomas Lejon \\ Centre for Telecommunications and Microelectronics \\ Victoria University, Melbourne-3011, Australia. \\ E-mail Kevin.tom1@research.vu.edu.au \\ * Ericsson AB, Business Unit Access, SE 16480, Sweden. \\ E-mail Thomas.lejon@ericsson.com
}

\begin{abstract}
Class-E amplifier has the potential to deliver high efficiency required for the next generation wireless systems. In this journal, we discuss a novel load pull analysis technique to characterize the efficiency performance of Class- $E$ amplifier in an outphasing power combining scheme. Class-E amplifier is not an ideal current or voltage source as is required for the traditional analysis of outphasing structures. It requires a phase modulated input signal and has a non-linear transfer characteristic which is a function of load impedance. Here we define an operating load locus based on the load pull analysis which can be used to predict the non-linear transfer function, efficiency, output power, input drive phase and many other factors associated with the outphasing class-E amplifier. This scheme could also be used to characterize any amplifier class in an outphasing structure. Finally modulation performance of Class-E amplifier using PWM technique is also presented.
\end{abstract}

\section{INTRODUCTION}

Wireless basestations will need to adapt to handle the new requirements of the next generation of wireless system (4G). Wider bandwidths, higher bit rates, multiple antenna structures and new bandwidth efficient orthogonal frequency division multiplexing (OFDM) modulation schemes are required [1-5]. Transceivers will need higher transmission powers, greater fidelity (reduced EVM - error vector magnitude) and greater integration. However they must fit into the same volume and require the same or less basestation air-conditioning load (or battery life for terminals). Heat dissipation must therefore be reduced, and this requires an increase in efficiency. The RF power amplifier (PA) dominates the efficiency performance.
Power amplifiers will play an important role in these next generation systems. This critical component dictates the size, cost and performance of the overall system. The main problem in power amplifier design is the efficiency. By improving the efficiency of a power amplifier less heat is dissipated in the device, this has many benefits. By reducing heat dissipation lower rated devices can be used for amplification and smaller heat sinks designed, this reduces the size of the overall system and increases its reliability, since heat is a major contributor of device failure. These size and reliability benefits, add up producing a cost effective solution.

There are two general types of PAs-linear PAs and switch mode PAs. Linear PAs like class-A and class-B output scaled versions of the input at the expense of efficiency. In contrast switch mode PAs offer the potential for better efficiency but are generally nonlinear. Recent advances in linearization techniques (Feed Forward [6], Pre-Distortion [7]) can overcome the latter deficiency.

Switch mode PAs run on one general principle of minimizing the power dissipated in the transistor. The ideal case would be if the transistor behaved as a switch. When the switch is open, no power is dissipated since there is no current flowing. When the switch is closed, no power is dissipated since there is no voltage across the switch. Since transistors are not perfect switches, the power can be minimized by making sure current and voltage are never high simultaneously. The efficiency of switch PAs varies with the output signal level which reduces their performance with modulated signals.

A tradeoff between efficiency and linearity always exists in PA design. Conducting class PAs such as class-A and class $A B$ offer good linearity but are inefficient with envelope varying signals. On the other hand switching class PAs such as class-D, E and $F$ 
have excellent efficiency but are very non linear [6]. A typical feed-forward class $A B$ power amplifier has efficiency in the range of $10 \%$ with modulated signals [7]. Alternatively a switch mode class-E amplifier attains drain efficiency of $45 \%$ when operated with $8 \mathrm{~dB}$ peak to average Rayleigh enveloped signal [8]. But they are highly non linear forcing the need for compensation in the modulator circuits. This journal looks at techniques to obtain the non-linear modulation characteristics which are optimized for power output and efficiency.

Linear amplification using non linear components (LINC) also known as outphasing [9-10] is one technique for obtaining linear operation from switched outputs, shown in Fig 1.

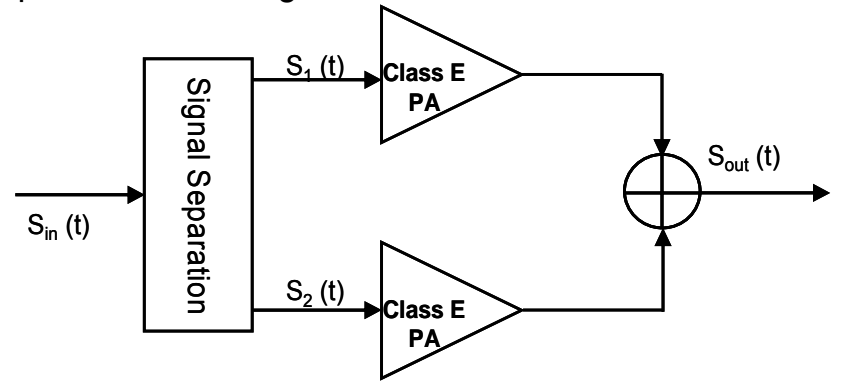

Figure 1. Outphasing Principle

Here an amplitude and phase modulated signal $S_{\text {in }}(t)=|S(t)| \exp (j \varphi(T))$ is decomposed into two constant amplitude signals $\left(S_{1}(t), S_{2}(t)\right)$ that can then be amplified using two highly efficient, non linear PAs, without adding any further distortion. The PA outputs are then summed to yield the signal for transmission $S_{\text {out }}(t) . \quad V_{0}$ denotes the amplitude of each branch signal and $\theta$ the input drive phase that determines the amplitude, $|\mathrm{S}(\mathrm{t})|$.

$$
\begin{aligned}
& S_{\text {in }}(t)=S(t) \cos [\omega t+\phi(t)] \\
& S_{1}(t)=V_{0} \cos [\omega t+\phi(t)+\theta(t)] \\
& S_{2}(t)=V_{0} \cos [\omega t+\phi(t)-\theta(t)] \\
& S_{\text {out }}(t)=S_{1}(t)+S_{2}(t)=2 V_{0} A(t) \cos [\omega t+\phi(t)]
\end{aligned}
$$

This scheme enables linear amplification of the original signal with system efficiency comparable to that of the used PAs. High efficiency of outphasing system is possible only if the PAs behave as ideal voltage sources. In such cases the efficiency of PAs is independent of the load impedance. Traditional LINC analysis using overdriven class- $B$, class- $C$ or class-D amplifiers assume a voltage source [11,12] for both amplifiers as this approximation is true. When it comes to the class-E amplifier it's not an ideal voltage or current source. Its output characteristic is a function of load impedance [13]. Therefore the traditional LINC analysis does not apply for class-E amplifiers instead we propose a load pull analysis method.

In practice saturated class B/C PAs cannot behaves as perfect voltage source when the load impedance goes reactive [12]. So this load pull analysis method is also applicable to any amplifier class used in an outphasing structure.

In this journal a load pull analysis of class- $E$ amplifier is performed to chararcterise its LINC performance. This is possible as LINC is a load modulation. Load modulation is a technique in which amplitude modulated signals are produced by the dynamic variation of the load impedance of the power amplifier. Section II discusses class-E amplifier operation. Section III examines outphasing power combining schemes. Section IV considers the load pull analysis of class-E amplifier using LINC power combining technique. Section $V$ discusses pulse width modulation performance of the Class-E amplifier.

\section{CLASS-E AMPLIFIER}

The basic topology of class-E amplifier is shown in Fig.2. The circuit includes a transistor operated as a switch, a shunt capacitor $\mathrm{C}_{1}$ which includes intrinsic transistor output capacitance $\left(\mathrm{C}_{\mathrm{ds}}\right)$, RF choke $\mathrm{L}_{1}$, a series tuned output circuit $L_{0} C_{0}$ and the load resistor, R.

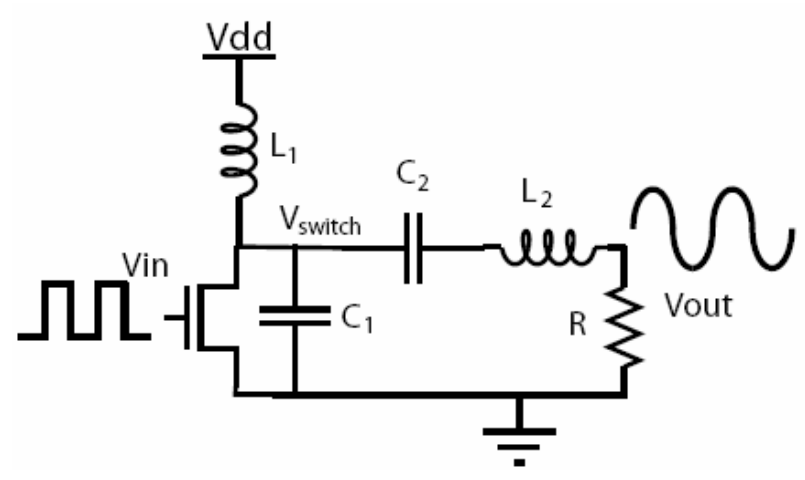

Figure 2. Class-E Amplifier

The class-E amplifier is a switch based power amplifier that achieves high efficiency at constant power output [13-17]. Theoretically it can achieve $100 \%$ efficiency. High efficiency can be obtained by keeping the voltage-current overlap across the transistor small. This is done using a technique called zero voltage switching (ZVS). This technique ensures that the voltage across the switch is near zero when the device turns on. This is achieved through a matching network consisting of a shunt capacitor and a series resonant tank. This matching network also 
sets the slope of the switch voltage to zero at turn on there by reducing the stress on the device. This is called zero derivative switching (ZDS). At the turn-off transition little power is lost as well. The shunt capacitor keeps the voltage low while the current shuts off. Most of the power loss occurs when the switch is on. The current through the device is large enough that with a small on resistance, the power loss is significant. A typical voltage and current waveform across the switch is shown in Fig-3. The switch voltage reaches zero with zero slope when the device turns on. (Time 77nsec in Figure-2)

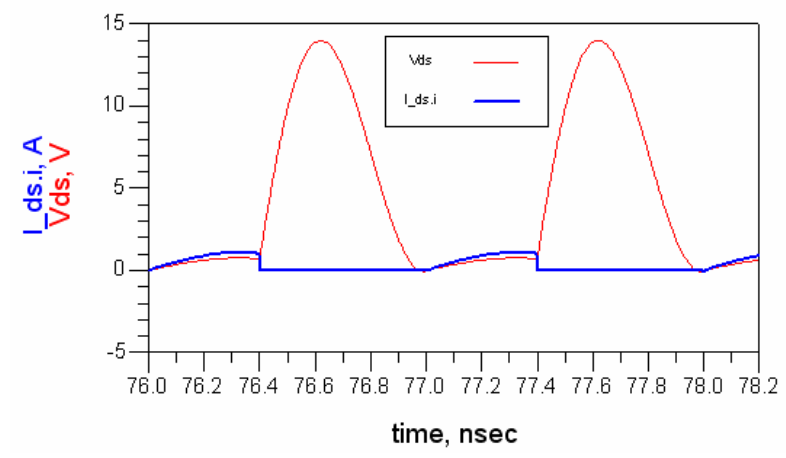

Figure 3. Current/Voltage Waveform across transistor

The design of class $\mathrm{E}$ power amplifier can be done analytically if the RF output is assumed sinusoidal (i.e. infinite loaded quality factor, $Q_{L}$ ). When the loaded $Q$ is not finite the solution cannot be derived analytically. This causes error in output power calculation [13]. This error gets larger as the loaded $Q$ becomes smaller. To account for the output power error from the loaded $Q$, design equations that take into account loaded $Q$ are used in this design [13].

$$
\begin{aligned}
& P=\frac{V c c^{2}}{R} 0.576801\left(1.0000086-\frac{0.014395}{Q L}-\frac{0.577501}{Q^{2} L}+\frac{0.205967}{Q^{3} L}\right) \\
& \mathrm{C}_{1}=\frac{1}{2 \pi \mathrm{fR}\left(\frac{\pi^{2}}{4}+1\right) \frac{\pi}{2}}\left(0.99866+\frac{0.91424}{\mathrm{QL}_{L}}-\frac{1.03175}{\mathrm{Q}^{2} \mathrm{~L}}\right)+\frac{0.6}{(2 \pi \mathrm{f})^{2} \mathrm{~L}_{1}} \\
& C_{2}=\frac{1}{2 \pi f R}\left(\frac{1}{Q_{L}-0.104823}\right)\left(1.00121+\frac{1.01468}{Q_{L}-1.7879}\right)-\frac{0.2}{(2 \pi f)^{2} L_{1}} \\
& L_{2}=\frac{Q_{L} R}{2 \pi f}
\end{aligned}
$$

The series resonant circuit $\mathrm{L}_{0} \mathrm{C}_{0}$, is usually not tuned to the operating frequency, $f$, having at this frequency a net series reactance $\mathrm{jX} \mathrm{L}=2 \pi \mathrm{f} \Delta \mathrm{L}$ (Fig. -4 ) produced by the difference in the reactances of the inductor and capacitor of the series tuned circuit given by Eq. (9).

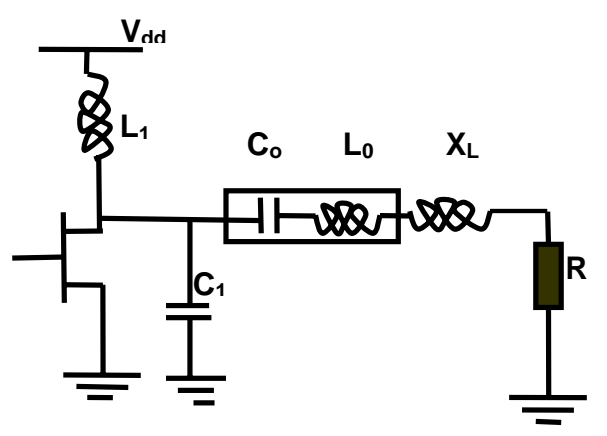

Figure 4. Class-E amplifier with residual load impedance

$$
\mathrm{XL}=2 \pi \mathrm{fL} 0-\frac{1}{2 \pi \mathrm{fC} 0}
$$

In this analysis $\mathrm{L}_{0} \mathrm{C}_{0}$ is considered as a resonant circuit tuned to the operating frequency $f$, in series with a net inductance $X_{L}$. This design uses Motorola (MRF9745T1) high frequency LDMOS FET. This model has an output capacitance of $11 \mathrm{pF}\left(\mathrm{C}_{1}\right)$, drain source resistance $\left(R_{d s}\right)$ of $1 \Omega$, switch breakdown voltage of $35 \mathrm{~V}$ and supply voltage of $5.8 \mathrm{~V}$. Selecting frequency (f) as $1 \mathrm{GHz}$ and $\mathrm{Q}$ to be 10 the resonant tank components $\mathrm{C}_{0}$, and $\mathrm{L}_{0}$ can be determined as given in [3] (Table 1). $R$ is taken as $3.5 \Omega$ to get an output power of 2.2W. RF choke (RFC) $L_{1}$ is selected to be $20 \mathrm{nH}$, which keeps the ac ripple less than $5 \%$. Simulations are done using Agilent ${ }^{\mathbb{Q}}$ ADS.

TABLE I. Class-E Component Values

\begin{tabular}{|c|l|}
\hline $\begin{array}{l}\text { Component } \\
\text { Name }\end{array}$ & Value \\
\hline $\mathrm{L}_{0}$ & $6.65 \mathrm{nH}$ \\
\hline $\mathrm{X}_{\mathrm{L}}$ & $0.826 \mathrm{nH}$ \\
\hline $\mathrm{C}_{1}$ & $11 \mathrm{pF}$ \\
\hline $\mathrm{C}_{0}$ & $3.81 \mathrm{pF}$ \\
\hline $\mathrm{R}$ & $3.5 \Omega$ \\
\hline $\mathrm{L}_{1}$ & $20 \mathrm{nH}$ \\
\hline
\end{tabular}

\section{OUtPHAsing POWER COMBINING SCHEMES}

In order to exploit the inherent efficiency benefit of the ouphasing system, a suitable power combining scheme needs to be analysed. A LINC power combiner using class-E amplifier is shown in Fig 5. 


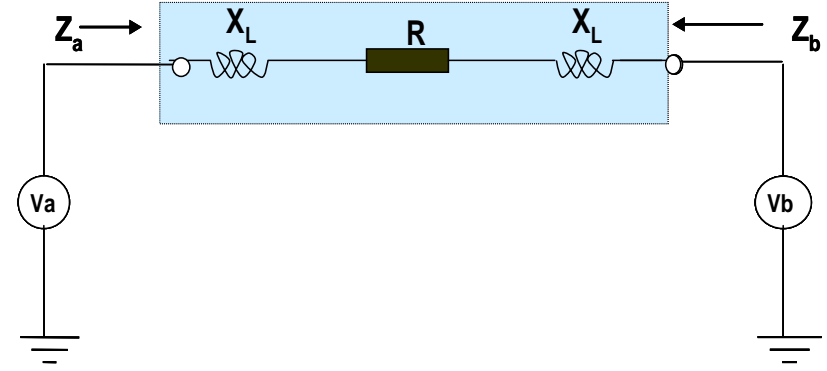

Figure 5. LINC power combiner using class-E

Here the class-E amplifiers are connected differentially to a common-mode load $\mathrm{R}$. The residual load reactance $X_{L}$ of each amplifier is considered as part of the power combiner network. This combiner network is linear. Za and Zb correspond to the effective load impedance seen by each amplifier while $\mathrm{Va}$ and $\mathrm{Vb}$ correspond to the output voltage of each amplifier for a given input drive phase (Ø). Both amplifiers are driven by square pulses having the same amplitude and frequency, but their phase is varied. The phase of $\mathrm{Va}$ and $\mathrm{Vb}$ is changed by varying the input drive phase $(\varnothing)$. When $\mathrm{Va}$ and $\mathrm{Vb}$ are in phase the amplifiers see infinite impedance and when they are $180^{\circ}$ out of phase they experience an effective load impedance of $R+j X_{L}$ resulting in class- $E$ operation of both amplifiers. There exists a unique relationship between $\left[Z_{a}, V_{a}\right]$ and $\left[Z_{b}, V_{b}\right]$ in terms of the power combiner circuit elements $R$ and $X_{L}$. This is obtained by solving the combiner network current equations and the solution is given in Eq. (10) and (11).

$$
\begin{array}{r}
V_{b}=\frac{\left[Z_{a}-R-j 2 X_{L}\right] V_{a}}{Z_{a}} \\
Z_{b}=-\left[Z_{a}-R-j 2 X_{L}\right]
\end{array}
$$

As $\mathrm{Za}$ is a function of the input drive phase (Ø), it can be seen that as $\varnothing$ changes the effective load impedance seen by each amplifier varies. This determines the efficiency performance of each amplifier in the LINC structure.

\section{CLASS-E LINC LOAD PULL ANALYSIS}

To get the operational load locus of the LINC combiner a load pull analysis of a class-E amplifier is performed to obtain a plot of $V_{a}$ and $Z_{a}\left(R+j X_{L}\right)$ Fig. 6 .

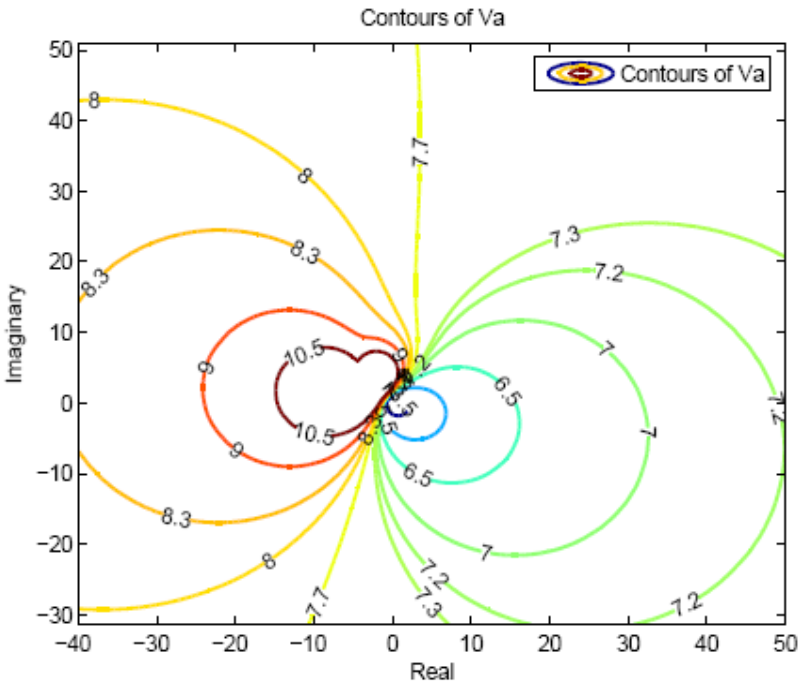

Figure 6. Load pull plot of single amplifier. Contours of Va vs. output load impedance (complex)
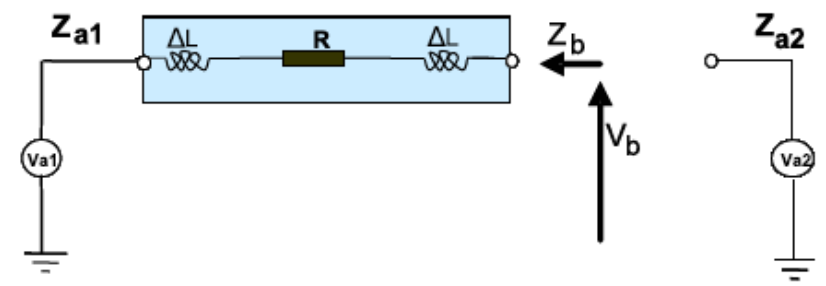

Figure 7. LINC Power Combiner Analysis

From Fig. 7 it can be noticed that for each value of $V_{a 1}$ and $Z_{a 1}$ there exists a unique value for $V_{b}$ and $Z_{b}$ seen from the other side of the combiner network, which is the load pull characteristic of amplifier $B\left[V_{b}\right.$, $\left.Z_{b}\right]$. $\left[V_{b}, Z_{b}\right]$ must be a valid combination of second amplifier $\left[\mathrm{V}_{\mathrm{a} 2}\right]$, which implies $\left[\mathrm{V}_{\mathrm{b}}, \mathrm{Z}_{\mathrm{b}}\right]$ is only valid when it equals $\left[V_{\mathrm{a} 2}, \mathrm{Z}_{\mathrm{a} 2}\right]$. Equilibrium is reached only when load pull seen at amplifier $B$ equals load pull at amplifier 2. Superimposing both plots shows the impedance where $\left[V_{b}, Z_{b}\right]$ equals $\left[V_{a 2}, Z_{a 2}\right]$, from which we obtain the operational load locus (Fig. 8) of the class-E amplifiers in the LINC combiner structure. This load locus is dependent on the operational frequency and changes with frequency of operation. 


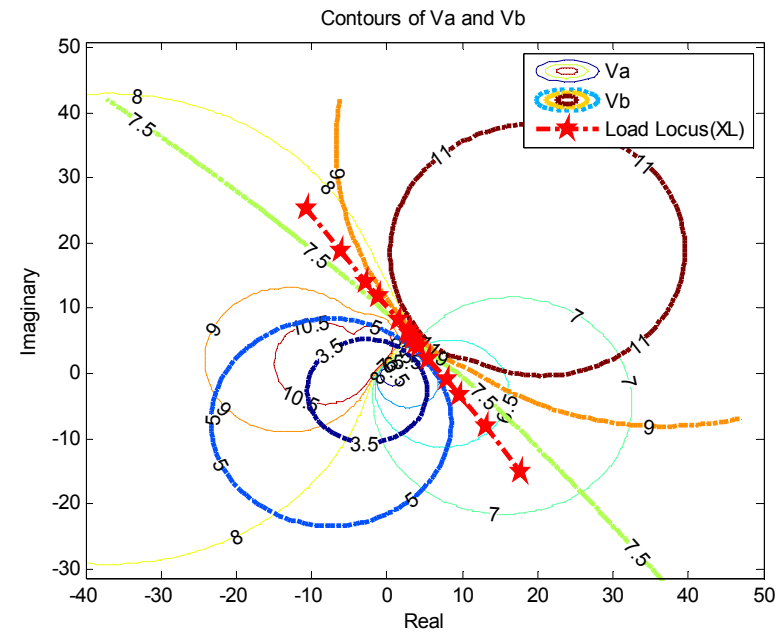

Figure 8. Class-E LINC load locus. Both amplifiers must operate on this line. The phase of the input drive signal defines the actual positions.

In Fig. 9 two new load lines are obtained by taking alternate values of $X_{L}$ in the combining network. These are superimposed on the output power contour of a single class- $E$ amplifier. $A_{1}$ and $B_{1}$ shows the operating points of both amplifiers in the LINC structure for a specific input drive phase. When one amplifier is operating at $A_{1}$ the amplifier at the other side of LINC will be at position $B_{1}$. So the total output power is obtained by adding the individual powers of each amplifier. Sometimes an amplifier sinks power depending on the drive phase.

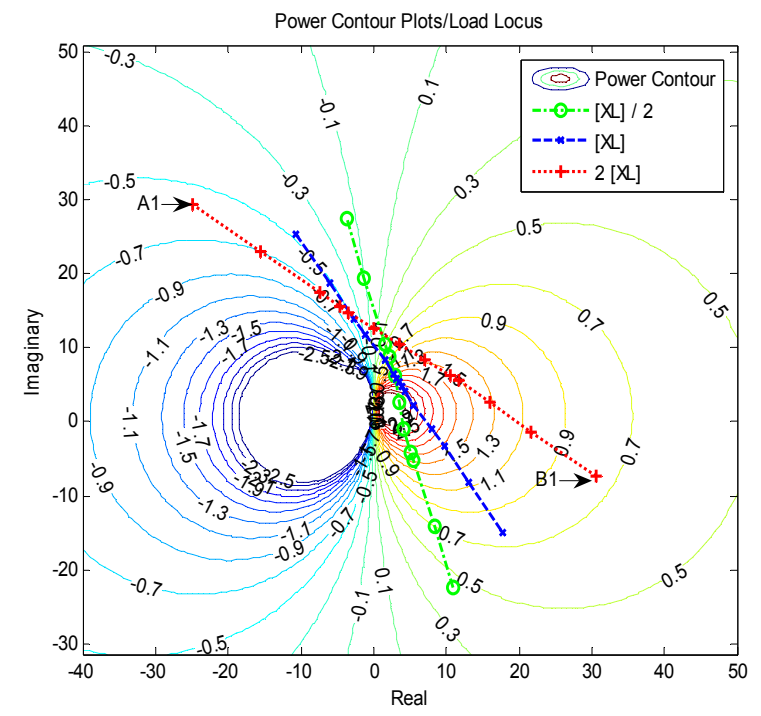

Figure 9. Amplifier output power contours as a function of load impedance. Output power is modulated by moving the amplifier operating point up and down the load locus.
Fig. 10 shows the plot of output power of Class-E LINC for different drive phases and $X_{L}$ values. Reducing $X_{L}$ increases output power. Output power is approximately linear with drive phase. Fig. 8 also shows the traditional cosine shape for an ideal saturated class B/C voltage source LINC system.

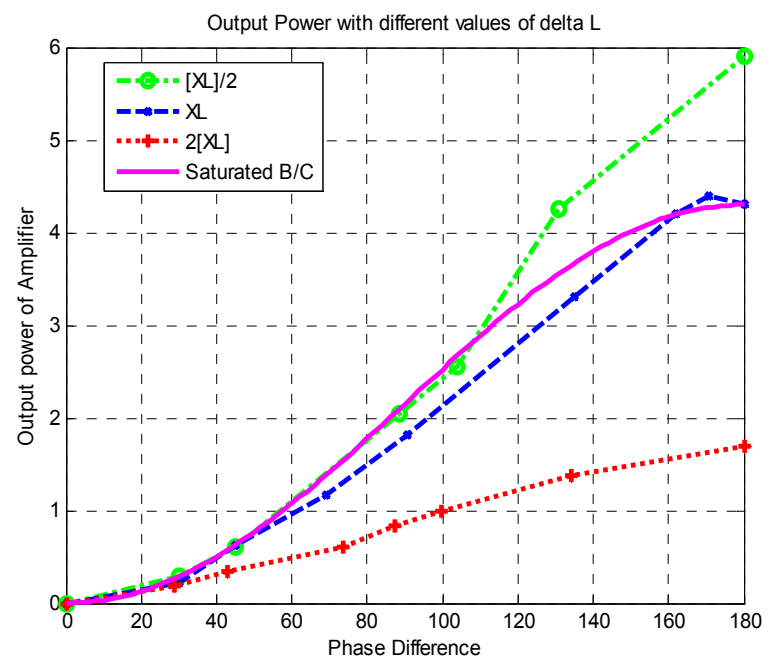

Figure 10. Output power of class-E LINC

Fig. 11 shows the power loss across the class-E LINC for different $X_{L}$ values and drive phases Losses in class-E amplifier are a combination of conduction and switching losses. In the optimal operation of class-E amplifier the only loss mechanism is conduction loss, which occurs in the LINC combiner at an input drive phase of $180^{\circ}$ (blue curve) in Fig. 11. For all other drive phases the loss in each amplifier is a combination of both losses and clearly dominated by switching losses as the amplifier moves away from zero-voltage switching (ZVS). Note the higher losses in $\mathrm{X}_{\mathrm{L}} / 2$ curve.

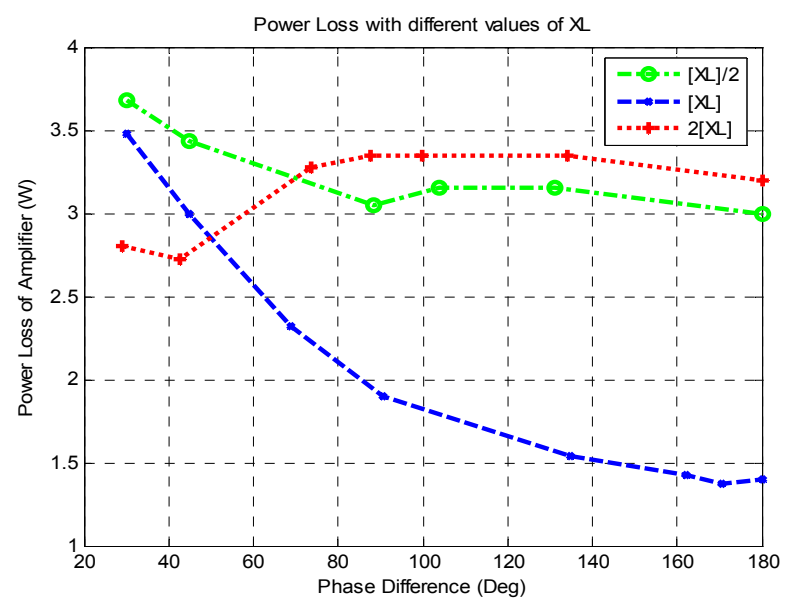

Figure 11 LINC 
In Fig. 12 output power is plotted against efficiency. Peak efficiency of $81 \%$ is obtained for the LINC combiner using $X_{L} / 2$ but the power is restricted to $1.8 \mathrm{~W}$. The plot gives a good indication of the performance of the Class -E LINC structure with various values of residual inductance $X_{L}$. A suitable $X_{L}$ could be selected based on the power probability distribution function of the modulation. Ideally, a structure with high efficiency and high output power is preferred, which indicates $X_{L}$ is the best choice.

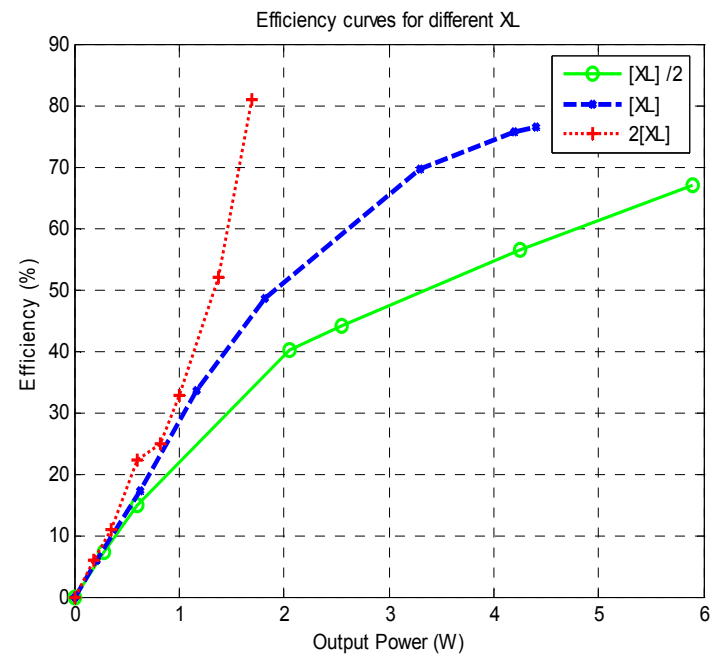

Figure 12. Efficiency vs. output power of class-E LINC for different $X_{L}$ values

\section{Pulse Width Modulated Class-E PoWer Amplifier}

Class-E amplifier achieves highest efficiency and constant output power when operated with a square wave having $50 \%$ duty cycle. By applying a pulse width modulated (PWM) input the output power can be varied. Simulating the design in ADS for different duty cycles results in the following waveforms, Fig. 13-14. Output power and efficiency at various duty cycles is plotted in Fig. 15.

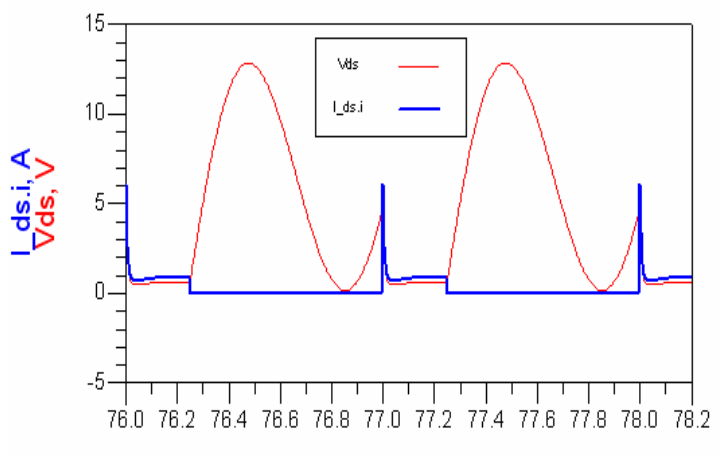

time, nsec

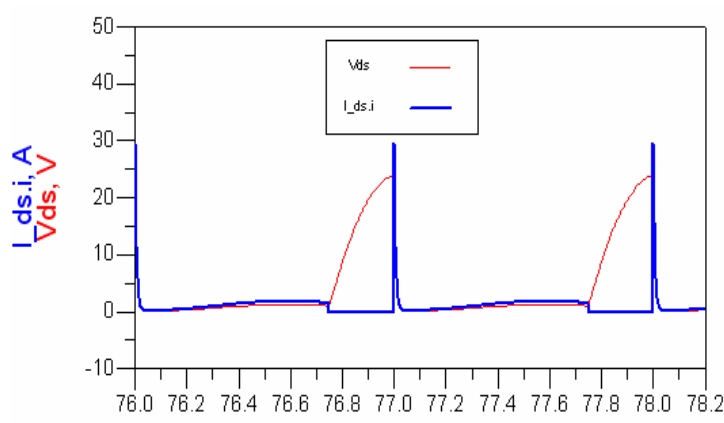

time, nsec

Figure 14. Switch waveform for $75 \%$ Duty Cycle
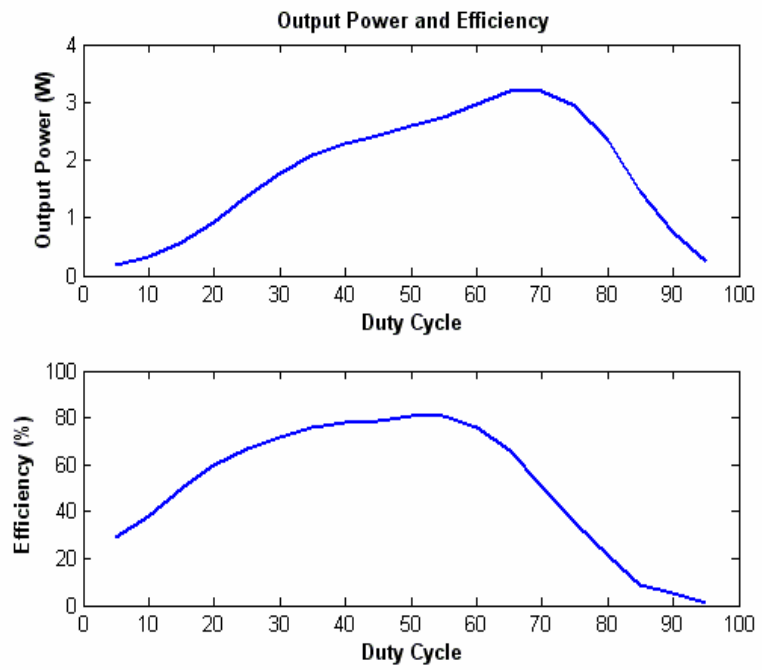

Figure 15 .

Output Power and Efficiency Variation with Duty Cycle

Output power is low for duty cycles less than $20 \%$ and more than $80 \%$. Efficiency is high for duty cycles around $50 \%$. At low duty cycles the RF choke has less time to store energy and at high duty cycles the stored energy is released not at the fundamental frequency. Tweaking the components $\mathrm{C}_{1}, \mathrm{C}_{2}$, and $\mathrm{L}_{2}$ the power and efficiency curves can be shifted towards lower or higher duty cycles.

The class $E$ power amplifier is designed for maximum efficiency when driven by a square wave with a $50 \%$ duty cycle. When the power amplifier is driven by a pulse width modulated square wave, the efficiency is expected to drop since ZVS is violated. Since the figure of merit is system efficiency, tweaking the system may result in better overall efficiency. Thus, tuning the matching network is explored in [19]. Perturbations are done on three components of the matching network - the shunt capacitor $\left(\mathrm{C}_{1}\right)$, resonant capacitor $\left(\mathrm{C}_{2)}\right.$ and resonant inductor $\left(\mathrm{L}_{2}\right)$.

Figure 13. Switch waveform for 25\% Duty Cycle 


\section{CONCLUSIONS}

In this paper we discussed a suitable scheme to characterize the LINC performance of class-E amplifier. Class-E amplifier is neither an ideal voltage nor a current source, so traditional LINC analysis does not apply to class-E amplifiers. Instead a load pull analysis is proposed to get its transfer characteristics and its efficiency performance. The Class-E amplifier is capable of achieving an efficiency of around $81 \%$ when operated in an outphasing scheme. In this mode of operation switching losses dominate and account for more than $60 \%$ of the total losses. This load-pull analysis technique can be utilized to characterize any amplifier class in an outphasing power combining scheme. Further pulse width modulated performance of class-E amplifier is also discussed. The amplifier achieves an efficiency of $80 \%$ when operated in PWM mode.

\section{REFERENCES}

[1] H. Yang, "A road to future broadband wireless access: MIMO-OFDM based air interface," IEEE comm. Mag., vol 43, pp 53-60, Jan. 2005.

[2] A. Diet, C. Berland, M. Villegas and G. Baudoin, "EER architecture specifications for OFDM Transmitter using a class $\mathrm{E}$ amplifier", IEEE Microwave and Wireless Components Letters, vol. 14 no. 8, pp. 389-391, August 2004.

[3] C. Berland, I. Hibon, J.F. Bercher, M. Villegas, D. Belot, D. Pache and V. le Goascoz, "A Transmitter Architecture for Nonconstant Envelope Modulation", IEEE Transactions on Circuits and Systems-II: Express Briefs, Vol. 53 no. 1, pp. 1317, January 2006.

[4] B.Berglund, J.Johansson, T.Lejon, Ericsson Radio Review,Available:

www.ericsson.com/ericsson/corpinfo/publications/r eview/2006 03/01.shtml

[5] F.H. Raab, P. Asbeck, S.Cripps, P.B. Kennington, Z. B. Popovic, N. Pothecary. J. F. Sevic and N.O. Sokal, "Power Amplifiers and transmitters for RF and microwave," IEEE Trans. Microw. Theory Tech., vol.50, no.3, pp. 326-334, Mar.2002.

[6] P.B. Kennington, "High Linearity RF Amplifier Design," Artech House, 2000.

[7] M. Faulkner, M. Mattsson, W. Yates. Adaptive Linearisation using Pre-Distortion. VTC 1991.

[8] K. Tom, M. Fauklner, T. Lejon, "Performance analysis of Pulse Width Modulated RF Class-E Power Amplifier," IEEE-VTC-S, pp 56-60, May 2006.

[9] H. Chireix, "High Power Outphasing Modulation," Proc. IRE, vol. 23, pp. 1370-1392, Nov.1935.

[10] D.C. Cox, "Linear amplification with nonlinear components," IEEE Trans. Commun., vol. COM23, pp. 1942-1945, Dec. 1974.
[11] Ilkka Hakala, Leila Gharavi, Risto Kaunisto, "Chireix Power Combining with Saturated Class-B Power Amplifiers," $12^{\text {th }}$ GAAS ${ }^{\circledR}$ Symposium, Amsterdam August 2004.

[12] Ilkka Hakala, David K. Choi, "A 2.14GHz Chireix Outphasing Transmitter," IEEE transactions on Microwave Theory and Techniques vol. 53, No. 6 June 2005.

[13] N. Sokal, "Class-E High Efficiency Power Amplifiers, from HF to Microwave," Microwave Symposium Digest June 1998.

[14] M. Kazimierczuk and K. Puczko, "Exact Analysis of Class-E Amplifier at any $Q$ and switch duty cycle," IEEE transactions on Circuits and Systems, February 1987.

[15] F. H. Raab, "Efficiency of outphasing RF poweramplifier systems," IEEE Trans. Commun. Vol. COM-33, pp. 1094-1099, Oct. 1985.

[16] F. H. Raab, "Idealized Operation of the Class E Tuned Power Amplifier," IEEE transactions on Circuits and Systems, vol. 24, No. 12, Dec 1977.

[17] F. H. Raab, "Effects of Circuit Variations on Class E Tuned Power Amplifier," IEEE journal JSSC, vol. Sc-13, No. 2, April 1978.

[18] "MRF9745T1 datasheet," Motorola, Available: www.chipdocs.com/pndecoder/datasheets/MOT/M RF9745T1.html

[19] K. Tom, M. Faulkner, "Performance analysis of RF class-E power amplifier with varying duty cycle", AtCRC Conference, pp 26-30, Nov-2005.

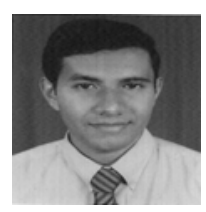

Kevin Tom received the B.Tech and M.Sc degrees in Electrical Engineering from Mahatma Gandhi University India and Linkoping University Sweden in 2000 and 2004 respectively. Currently he is working towards his $\mathrm{PhD}$ in Electrical Engineering from Victoria University Melbourne Australia.

He worked as Associate Consultant with Pentasoft Technologies, India from 2000-02. From 2003-04 he worked as Analog Design Engineer with TIFAC-CORE India. His research interests are in high-efficiency high-frequency switching mode tuned power amplifiers, RF Design and Analog IC design. 